\title{
Censo Brasileiro de Cirurgia Refrativa
}

\section{Brazilian Trends in Refractive Surgery}

Marcelo Vieira Netto ${ }^{1}$, Rodrigo França de Espíndola ${ }^{1}$, Rafael Garcia Fernandes Nogueira ${ }^{2}$, Mauro Campos ${ }^{3}$, Renato Ambrósio Jr. ${ }^{4}$, NeWton Leitão de Andrade ${ }^{5}$

\section{RESUMO}

Objetivo: Determinar preferências e práticas dos cirurgiões refrativos do Brasil. Métodos: Foi realizado um estudo transversal baseado na coleta de dados de um questionário aplicado durante o VI Congresso Brasileiro de Catarata e Cirurgia Refrativa em 2011. As questões também foram enviadas por e-mail aos membros dessa sociedade. Perguntas sobre preferências de técnicas, uso de novas tecnologias, volume cirúrgico, tipo de excimer laser, microcerátomo e topógrafos mais utilizados, uso de mitomicina C, colírios pós-operatórios, dentre outras, foram analisados.

Resultados: No total, 292 cirurgiões responderam a pesquisa. A maioria possui um volume mensal entre 2 a 4 olhos por semana $(57,60 \%)$. Grande parte $(64,50 \%)$ realiza tomografia de córnea de rotina e apenas $22,00 \%$ dos analisados não personalizam suas cirurgias. A técnica de ceratomileusis in situ a laser (LASIK) é a mais realizada e quando a ceratectomia fotorrefrativa (PRK) é utilizada, a maioria dos cirurgiões aplica a mitomicina C $(52,60 \%)$ nesses pacientes. A marca de excimer laser mais utilizada até o momento é a Nidek (26,12\%).

Conclusão: A técnica de LASIK é mais realizada pelos cirurgiões, sendo que a maioria personaliza parte de suas cirurgias e quando a ceratectomia fotorrefrativa é realizada, a mitomicina C é empregada pela maior parte dos entrevistados. A cirurgia bilateral é rotineiramente realizada pela maioria dos cirurgiões e o laser de femtosegundo ainda é empregado apenas por uma minoria dos cirurgiões.

Descritores: Miopia/cirurgia; Ceratomileuse assistida por excimer laser in situ; Ceratectomia fotorrefrativa; Mitomicina; Censos; Brasil

\begin{abstract}
Purpose: To determine preferences and practices of refractive surgeons in Brazil.

Methods: A cross-sectional study was conducted based on the data collected from a questionnaire applied during the VI Brazilian Congress of Cataract and Refractive Surgery and by e-mail sent to all members of that society. Refractive surgery techniques, use of emerging technologies, surgical volume, type of excimer laser and microkeratomes, mitomycin C, postoperative medications were analyzed among others questions.

Results: Two hundred ninety-two surgeons replied to the questions. The majority has a surgical volume between 2 and 4 eyes per week (57.60\%). Most of the surgeons (64.50\%) perform corneal tomography routinely and $22.00 \%$ of them do never customize their surgeries. The laser in situ keratomileusis (LASIK) is the main technique performed and when the photorefractive keratectomy (PRK) is applied; most of the surgeons uses mitomycin C (52.60\%) in these patients. The excimer laser of choice was the Nidek (26.12\%).

Conclusion: LASIK is the preferred surgical procedure and the majority customizes their refractive surgeries. When photorefractive keratectomy is performed, mitomycin Cis used by most of the surgeons (52.60\%). Bilateral surgery is routinely performed and the femtosecond laser is still used by few refractive surgeons.
\end{abstract}

Keywords: Myopia/surgery; Keratomileusis, laser in situ; Photorefractive keratectomy; Mitomycin; Censuses; Brazil

\section{INTRODUÇÃO}

A cirurgia refrativa está presente na prática clínica de profissionais de oftalmologia há pelo menos duas décadas. O advento do excimer laser e o aumento dos níveis de segurança e eficácia da cirurgia tornaram esta técnica um procedimento cirúrgico previsível e confiável ${ }^{(1)}$.

É estimado que aproximadamente 60 milhões de pessoas são candidatas à cirurgia refrativa nos Estados Unidos e que apenas 10\% do mercado americano é explorado(2).

A literatura nacional é carente de informações sobre preferências e práticas entre os cirurgiões refrativos. O presente estudo tem o objetivo de fornecer informações importantes sobre a cirurgia refrativa no Brasil. Questões como ablação personalizada, uso de tomógrafo de córnea, volume cirúrgico, preferências de técnicas cirúrgicas, colírios pós-operatórios, leito estromal residual entre outras, foram consideradas.

\section{MÉTODOS}

Realizou-se um estudo transversal durante o VI Congresso Brasileiro de Catarata e Cirurgia Refrativa de 2011. A pesquisa foi baseada na coleta dos dados de um questionário aplicado aos cirurgiões de refrativa participantes do evento. Ao término do congresso, o mesmo questionário foi enviado por e-mail aos membros da Sociedade Brasileira de Cirurgia Refrativa.

O questionário foi composto por 31 questões de múltiplas escoIha e por 1 questão aberta ("Qual porcentual de pacientes operados por você entre ceratomileusis in situ a laser (LASIK) e ceratectomia fotorrefrativa (PRK)?"). As seguintes variáveis foram estudadas: preferência de técnica cirúrgica; uso de mitomicina C; colírios pósoperatórios mais utilizados; tipo de excimer laser, microcerátomo, topógrafo e de tomógrafo de córnea; personalização de cirurgia; tipo de desepitelização usado na ceratectomia fotorrefrativa (PRK); leito

Submetido para publicação: 28 de outubro de 2011

Aceito para publicação: 15 de novembro de 2012

Trabalho realizado pela Sociedade Brasileira de Cirurgia Refrativa.

${ }^{1}$ Médico, Setor de Cirurgia Refrativa, Hospital das Clínicas, Faculdade de Medicina, Universidade de São Paulo - USP - São Paulo (SP), Brasil.

2 Médico, Departamento de Oftalmologia, Faculdade de Medicina, Universidade de São Paulo - USP 2 Médico, Departamento
São Paulo (SP), Brasil.

${ }^{3}$ Médico, Universidade Federal de São Paulo - UNIFESP - São Paulo (SP), Brasil.

${ }^{4}$ Médico, Rio de Janeiro (RJ), Brasil.

${ }^{5}$ Médico, Departamento de Cirurgia Refrativa do Hospital de Olhos Leiria de Andrade - Fortaleza

(CE), Brasil.

Financiamento: Não houve financiamento para este trabalho.

Divulgação de potenciais conflitos de interesse: M.Vieira Netto, Nenhum; R.F.Espíndola, Nenhum; R.G.F.Nogueira, Nenhum; M.Campos, Nenhum; R.Ambrósio Jr., Nenhum; N.L.Andrade, Nenhum. Endereço de correspondência: Rodrigo França de Espíndola. Praça das Hortências, 70. Condomínio Portal de Itu - Itu (SP) - 13301-689 - Brasil - E-mail: rodrigo166@uol.com.br 
estromal mínimo para ceratomileusis in situ a laser (LASIK); paquimetria mínima necessária para PRK e LASIK; medida intraoperatória do "flap" corneano; volume cirúrgico mensal, valor médio cobrado por cirurgia, bilateralidade, dentre outras.

A amostragem foi por conveniência (não probabilística), e o número de cirurgiões analisados foi estabelecido arbitrariamente de acordo com o número de voluntários participantes. A análise estatística baseou-se em dados descritivos da amostra, sendo as variáveis descritas por meio de médias ou proporções.

Durante o congresso, os questionários foram entregues aos participantes na entrada das sessões e recolhidos ao término da mesma. Os entrevistados foram orientados a não mudarem ou rasurarem suas respostas. Questões deixadas em branco, rasuradas e/ou assinaladas mais de uma vez foram desconsideradas para a análise estatística.

O mesmo questionário foi também enviado por e-mail a todos os membros da Sociedade Brasileira de Cirurgia Refrativa. As respostas foram enviados para o e-mail da mesma Sociedade.

Os questionários foram respondidos de forma anônima pelos cirurgiões participantes.

\section{RESULTADOS}

Responderam ao questionário 292 cirurgiões refrativos. Durante - VI Congresso Brasileiro de Cirurgia Refrativa foram analisadas as respostas de 245 oftalmologistas (83,90\%) e 47 (16,10\%) por e-mail enviados aos membros da Sociedade.

A maioria dos entrevistados realizam suas próprias cirurgias (89,70\%), possuem um volume cirúrgico mensal de 2 a 4 olhos por semana $(61,53 \%)$ e esse volume não vem aumentando nos últimos anos (52,30\%). O valor médio cobrado por olho está entre R\$1.000 e $R \$ 2.000(54,05 \%)$ e entre $R \$ 2.500$ e $R \$ 3.000$ (28,85\%).

\section{TÉCNICA CIRÚRGICA}

Com relação à preferência de técnica cirúrgica utilizada em pacientes com 30 anos de idade, com miopia de -10,00 D; espessura corneana maior que 550 micra e sem alterações topográficas, 42,47\% optam pela lente fácica, seguidos de LASIK $(30,97 \%)$ e PRK $(13,27 \%)$. Apenas 3,56\% dos entrevistados optaram pela extração do cristalino transparente e 9,73\% aguardariam melhores técnicas.

Quando o paciente é hipermétrope de +3,00 D com 45 anos de idade e curvatura e espessura coreanas normais, a maioria realizaria LASIK (70,64\%), seguido por extração do cristalino transparente (16,51\%), PRK $(9,17 \%)$ e 3,68\% aguardariam melhores técnicas. Se esse mesmo paciente hipermétrope possuísse $+5,00 \mathrm{D}$ a técnica preferida seria realizar a extração do cristalino transparente (39,47\%), seguido por LASIK (35,96\%), PRK $(5,26 \%)$ e apenas 2,63\% optariam pela lente fácica. Alguns dos analisados (16,68\%) nesta questão aguardariam melhores técnicas.

Dentre os questionários analisados, a técnica preferida para a correção de presbiopia em pacientes com cristalino transparente é a monovisão (53,33\%). Grande parte $(25,73 \%)$ não realiza cirurgias para a correção da pesbiopia, enquanto 18,09\% prefere a cirurgia da extração do cristalino transparente com implante de lente multifocal e apenas 2,95\% realizam a ablação multifocal com excimer laser.

Nas ablações de superfície, a técnica de rotina empregada por $58,00 \%$ dos cirurgiões é realizar o debridamento epitelial manual, seguido de debridamento com motor (22,40\%), debridamento por álcool (16,80\%) e Epi-LASIK (2,80\%).

Com relação ao uso da mitomicina $C$ no PRK, a maioria dos Cirurgiões utiliza em todos os pacientes $(52,60 \%)$. Os demais utilizam a mitomicina desta maneira: $22,80 \%$ usam em $2 / 3$ de seus pacientes; 9,60\% em metade dos casos; 8,70\% usam apenas para tratar "haze" pré-existente; $4,30 \%$ utilizam em 1/4 dos pacientes e apenas 1,70\% dos analisados nunca utilizam a mitomicina $C$.

Quanto à personalização da cirurgia refrativa, o maior porcentual de entrevistados (48\%) a realiza em 1/4 de seus pacientes, enquanto que $22 \%$ nunca personalizam suas cirurgias. Apenas 10\% dos entrevistados realizam a personalização em todos os casos, seguidos por $5 \%$ dos cirurgiões em 2/3 de seus casos e 15\% dos analisados em metade de seus pacientes.

O LASIK continua a ser a técnica cirúrgica de escolha dos cirurgiões refrativos. O gráfico 1 demonstra a distribuição de preferência entre LASIK e PRK.

A maioria dos analisados não realiza cirurgia refrativa em portadores de olho único (56,70\%). Sobre a lateralidade da cirurgia, a grande maioria prefere realizar o procedimento nos dois olhos simultaneamente tanto no LASIK (82,00\%), quanto no PRK $(70,70 \%)$.

Com relação à espessura corneana, 43,20\% cirurgiões assinalaram que o leito estromal residual na técnica de LASIK deve ser de 300 micra, seguido por 250 micra (32,40\%), 350 micra (23,40\%) e apenas $1,00 \%$ dos entrevistados não consideram leito estromal residual mínimo.

No LASIK, 60,20\% dos cirurgiões consideraram que a paquimetria mínima necessária para sua realização (considerando todos os demais parâmetros como normais) é de 500 micra, seguido por 520 micra (20,30\%), 490 micra (7,50\%), 480 micra (7,00\%), 450 micra (3,00\%) e apenas $2,00 \%$ dos analisados não utilizam limite mínimo. No caso do PRK, o maior porcentual dos respondentes considera o limite mínimo de 480 micra (30,60\%), seguido de 450 micra (23,40\%), 500 micra (20,00\%), 490 micra (19,00\%) e apenas 7,00\% não consideram nenhum limite mínimo.

A maioria não realiza a paquimetria intraoperatória $(85,30 \%)$ para medir a lamela corneana.

Com relação ao número de pacientes que evoluíram para ectasia após a cirurgia refrativa, a maioria dos entrevistados relataram não ter ciência de nenhum caso (58,30\%). O restante dos cirurgiões relataram ter ciência de caso(s) de ectasia(s) distribuídos da seguinte forma: 1 caso (18,00\%); 2 casos (9,00\%); 6 casos (4,60\%); 3 casos (3,70\%); mais de 10 casos (3,70\%); 4 casos (2,70\%).

\section{EXAMES PRÉ-OPERATÓRIOS E EQUIPAMENTOS CIRÚRGICOS}

De acordo com os cirurgiões participantes, os microcerátomos mais utilizados foram o Hansatome/XP (55,88\%) e o Moria (21,58\%). Apenas 4,98\% dos entrevistados realizam a confecção da lamela corneana com o laser de femtosegundo como demonstrado no gráfico 2.

Com relação às marcas de excimer laser mais utilizadas o Nidek (26,14\%), o Baush \& Lomb Z100 (22,53\%) e o Schwind Amaris (18,02\%) foram os mais citados. A distribuição das marcas de excimer laser citados no questionário está demonstrada no gráfico 3.

O modelo de topógrafo de córnea mais utilizado foi o EyeSys (34,69\%), seguido pelo Tomey (15,30\%) e Alcon Eye Map (9,18\%). O gráfico 4 demonstra a relação dos topógrafos mais citados.

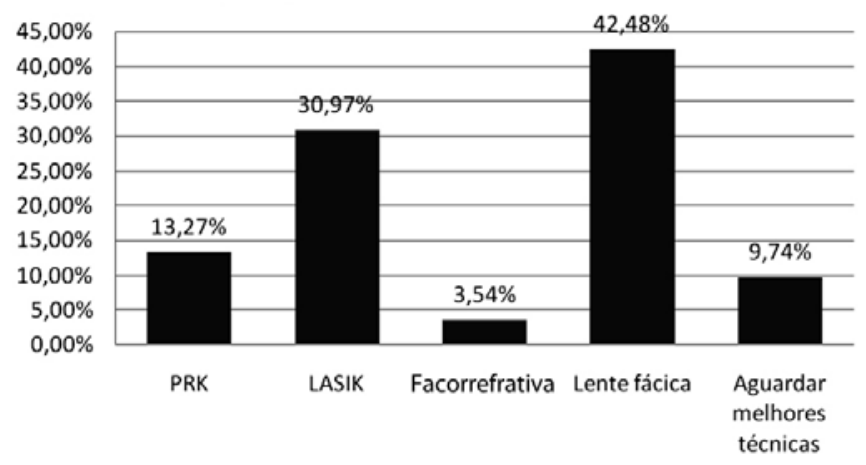

Gráfico 1. Procedimento refrativo de escolha para paciente de 30 anos, míope (-10,00 DE) com córnea $>550$ micra e sem sinais de ceratocone. 


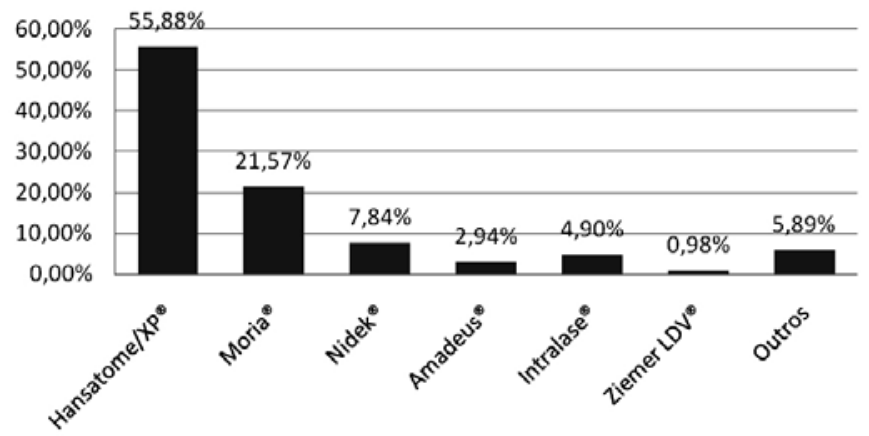

Gráfico 2. Distribuição dos microcerátomos mais utilizados entre os cirurgiões.

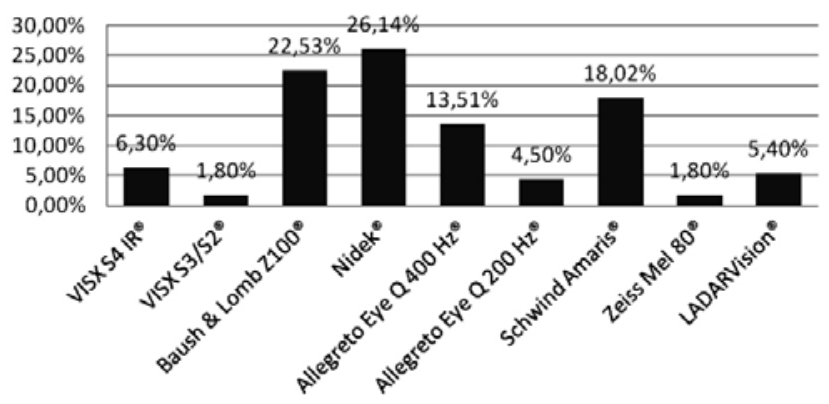

Gráfico 3. Distribuição dos excimer lasers mais utilizados entre os cirurgiões.

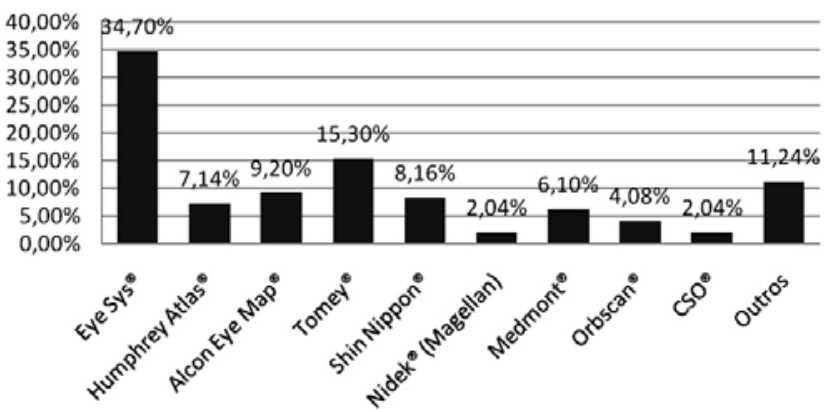

Gráfico 4. Distribuição dos topógrafos corneanos mais utilizados entre os cirurgiões.

A maioria dos entrevistados $(64,50 \%)$ utiliza tomógrafo de córnea rotineiramente, 32,00\% apenas em casos selecionados e 3,5\% nunca utilizam esse exame. O tomógrafo mais usado foi o Orbscan (60,00\%), seguidos pelo Pentacam (33,40\%) e o Galilei (6,60\%).

Os cirurgiões analisados não pretendem trocar ou adquirir um excimer laser nos próximos 12 meses $(71,68 \%)$ e a grande maioria $(75,67 \%)$ não pretende trocar ou comprar um laser de femtosegundo nos próximos 12 meses.

\section{Colírios Pós-operatórios}

O antibiótico mais prescrito foi o moxifloxacino (46,82\%) seguidos pelo gatifloxacino $(45,68 \%)$, ciprofloxacino $(3,00 \%)$, ofloxacino $(2,25 \%)$ e tobramicina $(2,25 \%)$.
O anti-inflamatório mais usado foi o acetato de prednisolona $(61,53 \%)$ seguido pela dexametasona (37,50\%) e loteprolol (0,97\%). A maioria dos entrevistados prescreve como anti-inflamatório não hormonal o cetorolac de trometamina $(64,76 \%)$, seguido pelo nepafenaco $(26,66 \%)$ e diclofenaco $(0,95 \%)$. Não utilizam esse medicamento $7,63 \%$ dos analisados.

\section{DISCUSSÃO}

O objetivo principal deste estudo é compartilhar e tornar disponíveis informações sobre as preferências e práticas em cirurgia refrativa no Brasil para todos os oftalmologistas, bem como identificar tendências entre os cirurgiões refrativos.

O LASIK é a técnica mais utilizada pelos cirurgiões entrevistados. A cirurgia bilateral simultânea é rotineiramente realizada pelos cirurgiões tanto nas técnicas de LASIK ou PRK. Esses resultados assemelham-se aos últimos levantamentos da Sociedade Americana de Catarata e Cirurgia Refrativa (ASCRS) ${ }^{(3,4)}$

O último censo brasileiro de cirurgia refrativa realizado no Brasil em 2005(5), aponta resultados semelhantes ao presente estudo. A maioria dos cirurgiões realizavam como técnica de escolha o LASIK, operavam os dois olhos simultaneamente, usavam o excimer laser da Nidek (42,60\% versus $26,14 \%$ em 2011) e o Hansatome era o microcerátomo mais utilizado (57,70\% versus 55,88\% em 2011$)$.

Porém algumas diferenças entre os dois censos foram notadas. Em 2005(5), 62,00\% não usavam anti-inflamatórios não hormonais contra apenas 7,63\% em 2011. Quarenta por cento dos entrevistados não realizavam a monovisão e no presente estudo, pouco mais de $50,00 \%$ preferiam esta técnica para a correção da presbiopia. O custo por cirurgia refrativa também variou entre os dois levantamentos, em 2005 a maioria cobrava até US\$ 500/olho (67,40\%) e hoje o valor cobrado (em dólares) por 54,05\% dos entrevistados varia entre US\$ 535 - US\$1070/olho.

Com relação à cirurgia personalizada, apenas 22\% dos analisados nunca realizaram essa modalidade em seus pacientes. Somente 10\% dos cirurgiões utiliza a cirurgia personalizada em todos os pacientes. Alguns autores ${ }^{(3)}$ relataram que os cirurgiões americanos indicam a cirurgia personalizada com maior frequência e cobram em média US\$ 300 a mais por olho com essa modalidade técnica, Em 2005, apenas 4,50\% dos cirurgiões brasileiros realizavam "wavefront"(5).

Apesar da cirurgia de retirada do cristalino transparente não ser regulamentada pelo Conselho Federal de Medicina no Brasil, ela foi considerada para correção de altas ametropias por boa parte dos entrevistados, tanto para correção de presbiopia como para hipermetropia de +5,00 D. E o número de cirurgiões que consideram esta técnica parece estar crescendo no Brasil, em 2005, a grande maioria dos cirurgiões $(61,50 \%)$ não realiza esse procedimento ${ }^{(5)}$.

Outra tendência que pode ser notada ao compararmos o censo anterior com os dados deste estudo é um aumento da indicação do PRK. Em 2005, praticamente 64\% dos cirurgiões não faziam ou realizavam até $10 \%$ de PRK considerando o volume cirúrgico total(5). Os dados deste estudo mostram que esse número caiu para aproximadamente 36\% em 2011. Esse aumento da frequência do PRK pode ser atribuído a uma maior preocupação com a ectasia pós-operatória, avanços na tecnologia dos lasers, uso da mitomicina C e diminuição da dor no pós-operatório com melhores anti-inflamatórias não hormonais.

Outros estudos também tem demonstrado um aumento na indicação do PRK, já que essa técnica não apresenta complicações na confecção da lamela corneana, possuí menor risco de ectasia pósoperatória e resultados comparáveis ao $\operatorname{LASIK}^{(6-9)}$.

Apenas 1,70\% dos cirurgiões nunca utilizam a mitomicina $C$ após o PRK, porém a maioria a utiliza rotineiramente, sendo que $52,60 \%$ dos cirurgiões a utilizam em todos os seus pacientes. Dados americanos apontam também para um crescente aumento no uso da mitomicina C (58,4\% em 2004 contra 49,50\% em 2002) $)^{(4)}$. 
Com relação ao laser de femtosegundo, somente uma pequena parte dos entrevistados $(4,90 \%)$ utiliza essa tecnologia e a grande maioria dos cirurgiões $(75,67 \%)$ não pretende trocar ou adquirir um laser de femtosegundo nos próximos 12 meses. O uso do IntraLase $^{\circledR}$ para a confeção da lamela aumenta ao redor do mundo e segundo Sandoval e colaboradores $5,10 \%$ dos cirurgiões utilizam essa tecnologia(4).

Apesar da importância epidemiológica, o presente estudo apresenta algumas limitações. A opção por questões de múltipla escolha facilita a análise, porém não leva em consideração outros tipos de respostas não listadas nas alternativas. Devido ao caráter anônimo e voluntário da pesquisa, é impossível compararmos as respostas daqueles que não enviaram o questionário para a análise. E por fim, o questionário foi enviado somente aos membros da Sociedade e aos participantes do VI Congresso Brasileiro de Catarata e Cirurgia Refrativa, e não para todos os cirurgiões que realizam cirurgia refrativa no Brasil. De uma forma geral, a análise fica restrita aos cirurgiões refrativos que participam dos congressos ou que fazem parte da Sociedade Brasileira de Cirurgia Refrativa.

Sabemos que os resultados apontados por esta pesquisa representam apenas as práticas de um determinado grupo de oftalmologistas em um determinado período de tempo. Práticas e tendências mudam com o passar dos anos, novas tecnologias e novas pesquisas são incorporadas rapidamente na prática diária dos cirurgiões ao redor do mundo. Por isso, levantamentos como este devem ser sempre estimulados e repetidos ao longo dos anos, abrangendo um número cada vez maior de cirurgiões.

\section{CONCLUSÃO}

O LASIK continua sendo a técnica mais utilizada pelos cirurgiões refrativos, apesar do aumento do emprego da técnica de PRK nos últimos cinco anos. A cirurgia bilateral é realizada rotineiramente pela maioria dos cirurgiões tanto nas técnicas de LASIK ou PRK.
A tomografia de córnea vem sento utilizada de forma rotineira pela maioria dos cirurgiões refrativos. Porém, o laser de femtosegundo ainda é empregado pela menor parte dos cirurgiões. O volume de cirurgias refrativas realizadas no Brasil não aumentou nos últimos anos, segundo os cirurgiões participantes.

\section{REFERÊNCIAS}

1. Bechara SJ, Garcia R, Medeiros FW, Barreto-Junior J, Netto MV. Cirurgia Refrativa. São Paulo: Artmed; 2009

2. Kuo IC. Trends in refractive surgery at an academic center: 2007-2009. BMC Ophthalmol [Internet]. 2011[cited 2012 Jun 21];11:11. Available from: http://www.ncbi.nlm. nih.gov/pmc/articles/PMC3115925/

3. Duffey RJ, Leaming D. Trends in refractive surgery in the United States. J Cataract Refract Surg [Internet]. 2004[cited 2010 Mar 19];30(8):1781-5. Available from: http:// www.sciencedirect.com/science/article/pii/S0886335004005784

4. Sandoval HP, Castro LE, Vroman DT, Solomon KD. Refractive Surgery Survey 2004. Cataract Refract Surg [Internet]. 2005[cited 2010 Jan 21];31(1):221-33. Available from: http://www.sciencedirect.com/science/article/pii/S0886335004010958

5. Victor G, Urbano A, Marçal S, Porto R, Francesconi CM, Forseto AS, et al. Primeiro censo brasileiro em cirurgia refrativa. Arq Bras Oftalmol [Internet]. 2005 [cited 2010 Oct 15];68(6):727-33.Available from: http://www.scielo.br/pdf/abo/v68n6/a05v68n6.pdf

6. Moshirfar M, Schliesser JA, Chang JC, Oberg TJ, Mifflin MD, Townley R, et al. Visual outcomes after wavefront guided photorefractive keratectomy and wavefront guided laser in situ keratomileusis: Prospective comparison. J Cataract Refract Surg [Internet]. 2010 [cited 2012 Nov 21];36(8):1336-43. Available from: http://www.sciencedirect. com/science/article/pii/S0886335010006930

7. Shortt AJ, Allan BD. Photorefractive keratectomy (PRK) versus laser-assisted in-situ keratomileusis (LASIK) for myopia. Cochrane Database Syst Rev. 2006;19(2):CD005135. Update in: Cochrane Database Syst Rev. 2013;1:CD005135.

8. Sugar A, Rapuano CJ, Culbertson WW, Huang D, Varley GA, Agapitos PJ, et al. Laser in situ keratomileusis for myopia and astigmatism: safety and efficacy: a report by the American Academy of Ophthalmology. Ophthalmology [Internet]. 2002 [cited 2012 Set 12];109(1):175-87. Available from: http://www.sciencedirect.com/science/article/ pii/S0161642001009666

9. Forseto AS, Nosé RA, Nosé W. PRK versus LASIK para correção de miopia baixa e moderada. Arq Bras Oftalmol [Internet]. 2000[citado 2010 Dez 21];63(4):257-62. Disponível em: http://www.scielo.br/pdf/abo/v63n4/12327.pdf 